\title{
Allelic heterogeneity
}

National Cancer Institute ( $\mathrm{NCl})$

\section{Source}

National Cancer Institute (NCI). Allelic heterogeneity.

Different mutations in the same gene that cause different phenotypic manifestations or severity of disease. 